CALT-68-2156

hep-ph/9802335

DOE RESEARCH AND

DEVELOPMENT REPORT

\title{
Diffusion and Decoherence of Squarks and Quarks During the Electroweak Phase Transition
}

\author{
Hooman Davoudiasl ${ }^{*}$ and Eric Westphal ${ }^{\dagger}$ \\ California Institute of Technology, Pasadena, CA 91125 USA
}

\begin{abstract}
To estimate the diffusion constant $D$ of particles in a plasma, we develop a method that is based on the mean free path $\lambda$ for scatterings with momentum transfer $q \gtrsim T$. Using this method, we estimate $\lambda$ and $D$ for squarks and quarks during the electroweak phase transition. Assuming that Debye and magnetic screening lengths provide suitable infrared cutoffs, our calculations yield $\lambda \lesssim 18 / T$ and $D \lesssim 5 / T$ for both squarks and quarks. Our estimate of $\lambda$ suggests that suppressions of charge transport due to decoherence of these strongly interacting particles during the electroweak phase transition are not severe and that these particles may contribute significantly to electroweak baryogenesis.
\end{abstract}

${ }^{*}$ E-mail address: hooman@theory.caltech.edu

†E-mail address: westphal@theory.caltech.edu 


\section{INTRODUCTION}

In recent years, many scenarios incorporating extensions of the Minimal Standard Model (MSM) have been proposed for the generation of the Baryon Asymmetry of the Universe during the electroweak phase transition. A much studied mechanism uses the transport of some quantum number, via the $\mathrm{CP}$ violating interactions of certain particles with the expanding broken phase Higgs wall, into the unbroken phase during a first order electroweak phase transition. This transported quantum number, such as the axial top quark number, then biases the equilibrium in the direction of baryon number violation, ultimately generating a baryon asymmetry through weak sphaleron processes [1,2]. Of the extensions of the MSM used in these scenarios, those based on supersymmetric models such as the Minimal Supersymmetric Standard Model (MSSM) are the best motivated. By including supersymmetric particles, extra CP violation can be naturally introduced into a mechanism for baryogenesis. It is argued that a strongly first order phase transition can be achieved if the broken phase right-handed stop mass is less than, or of the order of, the top quark mass [3,4]. In this case, squarks with soft supersymmetry breaking masses $m_{s} \sim T$, where $T \sim 100 \mathrm{GeV}$ is the temperature of the plasma, could have an important role in the charge transport mechanism [5]. However, to study charge transport via stops, one needs to have an estimate of their diffusion properties in a plasma at the electroweak scale.

In Refs. [6, [7, a set of approximations in conjunction with the Boltzmann equation for quarks in the plasma of MSM particles were used to estimate the quark diffusion constant $D_{q}$. As strong interactions dominate the diffusion process, and since stops are strongly interacting particles, it has been assumed that the estimate $D_{q} \sim 6 / T$ of Ref. [6] is applicable to stops as well (the validity of this assumption is not a priori obvious because of the different statistics, masses, and couplings of squarks and quarks). This estimate is derived using an approximate gluon propagator with the thermal mass of the longitudinal gluons $m_{g}$ as an infrared cutoff and ignoring the different thermal properties of the transverse and longitudinal gluons. The use of the approximate gluon propagator can only yield the leading-logarithmic behavior 
and does not result in the correct leading $\alpha_{s}^{2}$ non-logarithmic contribution [6,7]. However, the diffusive process is expected to be dominated by the $t$-channel gluon exchange diagrams, and for these diagrams, the leading logarithm contribution is expected to be dominant [6]. A more comprehensive treatment in Ref. [7] gives $D_{q} \sim 3 / T$. Again, this estimate is at the level of the leading logarithm.

To study the diffusion of particles in a plasma, one must consider scattering processes in which the momentum transfer $q$ is not small. For the $t$-channel processes we consider in this paper, we approximate this effect by an infrared regularization of $q$ such that $q \gtrsim T$, since the typical momenta of the scattering particles are of order $T$. That is, we assume that such a transfer of momentum in the scattering process randomizes the momentum of the diffusing particle, as an approximation of the physics invovled. This momentum randomization approximation is implemented naturally by using as cutoffs the longitudinal and transverse thermal masses of the exchanged gluons which are comparable in magnitude to the temperature $T$ of the plasma. We note here that the thermal masses of gluons depend parametrically on the strong coupling constant $g_{s}$, and that these masses are comparable to the temperature only for realistic values of $g_{s} \sim 1$. If we take the limit in which $g_{s} \ll 1$, these masses will be small compared to the temeprature $T$, and cannot be used as cutoffs in our approach. In this paper, we use the above momentum randomization approximation to calculate the elastic mean free path $\lambda$ associated with the diffusive processes (for which $q \gtrsim T)$ and relate it to the diffusion constant $D$. Henceforth, the words "mean free path" refer to this diffusive mean free path.

In electroweak baryogenesis scenarios that use charge transport, the $\mathrm{CP}$ violating interactions of the charge carriers with an expanding Higgs wall eventually result in the generation of baryon number. However, within the Higgs wall, multiple scatterings in which the final

\footnotetext{
${ }^{*}$ Non-elastic processes in which the species of the particle changes do contribute to the mean free path; however, these are not considered to be diffusive processes here.
} 
momentum of the charge carrier differs significantly from its initial momentum wash out the asymmetry caused by CP violation and suppress baryogenesis. This effect is known as decoherence. These same processes also contribute to the diffusion of the particles within the plasma. Therefore, the diffusive mean free path $\lambda$ which we calculate is a relevant parameter for estimating the supression due to decoherence.

In this paper, we use the method described above to estimate the mean free path $\lambda_{s}$ and the diffusion constant $D_{s}$ of stops that have a soft supersymmetry breaking mass $m_{s} \sim T$ in the unbroken phase of the electroweak plasma. We use the same method to estimate the mean free path $\lambda_{q}$ and the diffusion constant $D_{q}$ for quarks and compare our values with those of Ref. [6]. We find that our method reproduces the results of Ref. [6] for the set of parameters used therein. In general, our results suggest that the values of $\lambda$ and $D$ of squarks are close to those of quarks.

In calculating $\lambda$ and $D$, we consider only strong interactions, for they dominate the diffusion of squarks and quarks in the plasma. In the case of squarks, we further assume that scatterings from and via the heavy gluinos and squarks do not contribute significantly, leaving only quarks and gluons as the dominant scatterers and mediators. As consideration of more scatterers can only decrease the calculated values of $\lambda$ and therefore $D$, the inclusion of only quark scatterers will yield an upper bound (and even a reasonable order of magnitude estimate) for the size of the squark diffusion constant $D_{s}$, in light of the results of Ref. [7]. In computing $D_{q}$, to facilitate comparison, we follow Ref. [6] and only consider $t$-channel quark-quark scattering.

As explained above, we implement our momentum randomization approximation using the longitudinal and transverse gluons thermal masses $m_{g}$ and $m_{t}$ in the plasma, referred to as Debye and magnetic masses, respectively, as physical infrared cutoffs for the exchanged gluon momentum. Since these masses depend on $g_{s}$, the momentum transfer $q$ in our calculations is parametrically of order $g_{s} T$, and the use of Hard Thermal Loop (HTL) propagator for the gluons is valid [8,9]. In the limit $g_{s} \ll 1$, this corresponds to small momentum transfer. However, we note that in our approximation, we demand $q \gtrsim T$. Thus, in the $g_{s} \ll 1$ 
limit, in our approach, we have to abandon the thermal masses as cutoffs, and choose a cutoff $q_{c u t}$ parametrically independent of $g_{s}$, and such that $q_{c u t} \gtrsim T$. Then, in this technical sense, in our approach, the use of the HTL gluon propagator would not be justified, since the momentum carried by the exchanged gluon would not be parametrically soft, that is $\mathcal{O}\left(g_{s} T\right)$, as required by the HTL formalism [8,9]. Our method does not yield the leading logarithm behavior, as $g_{s} \rightarrow 0$, obtained in Refs. [6,7] for the $t$-channel processes considered therein. Nonetheless, for the physical values of $g_{s}$ at the electroweak phase transition, we obtain similar results, suggesting that our scheme reasonably approximates the physics. For realistic values of $g_{s}$, we use the gluon thermal masses as cutoffs in our momentum randomization approximation, since they naturally arise in the plasma. Then, parametrically, we are allowed to use the HTL propagator for the gluon. In this work, to incorporate thermal effects qualitatively, we approximate the effect of the HTL propagator by separating the gluon propagator into transverse and longitudinal parts that in general have different thermal masses. Whereas $m_{g}$ is calculable at one loop, $m_{t}$ is not calculable perturbatively and is unknown. Therefore, we will present our results for two representative values of $m_{t}$.

In the next section, we describe our method for calculating the diffusion constant of particles in the plasma. In Section III, we present our estimates for $\lambda_{s}, D_{s}, \lambda_{q}$, and $D_{q}$, followed by a discussion of our results. The appendix contains some information on the approximate thermal gluon propagator we use in our calculations.

\section{CALCULATION OF THE MEAN FREE PATH AND THE DIFFUSION CONSTANT}

Let us consider a two body scattering process where the initial and final particles have 4-momenta $(p, k)$ and $\left(p^{\prime}, k^{\prime}\right)$, respectively. We refer to each particle by its 4 -momentum for the rest of this section. The $p$-particle, whose diffusion constant we calculate, scatters from the $k$-particle. For processes relevant to the calculation of the diffusion constant $D$, the final state $p^{\prime}$-particle is of the same species as the initial $p$-particle. The $p$-particle, $k$-particle, 
$p^{\prime}$-particle, and $k^{\prime}$-particle have thermal distributions $\rho_{p}, \rho_{k}, \rho_{p^{\prime}}$, and $\rho_{k^{\prime}}$, respectively. The density per unit volume of a particle with 4 -momentum $p$ is given by $\rho_{p} d^{3} p /(2 \pi)^{3}$.

The transition probability for the above process per unit volume and per unit time is

$$
\begin{gathered}
\eta=\frac{d^{3} p}{(2 \pi)^{3} 2 p^{0}} \frac{d^{3} k}{(2 \pi)^{3} 2 k^{0}} \frac{d^{3} p^{\prime}}{(2 \pi)^{3} 2 p^{\prime 0}} \frac{d^{3} k^{\prime}}{(2 \pi)^{3} 2 k^{\prime 0}}(2 \pi)^{4} \delta^{(4)}\left(p+k-p^{\prime}-k^{\prime}\right)|\mathcal{M}|^{2} \\
\times \rho_{p} \rho_{k}\left(1 \pm \rho_{p^{\prime}}\right)\left(1 \pm \rho_{k^{\prime}}\right),
\end{gathered}
$$

where $\mathcal{M}$ is the amplitude for the scattering, and \pm is for final state bosons or fermions, respectively. Let $d \sigma$ be the differential cross section for this process,

$$
d \sigma=\frac{d^{3} p^{\prime}}{(2 \pi)^{3} 2 p^{\prime 0}} \frac{d^{3} k^{\prime}}{(2 \pi)^{3} 2 k^{\prime 0}}(2 \pi)^{4} \delta^{(4)}\left(p+k-p^{\prime}-k^{\prime}\right) \frac{|\mathcal{M}|^{2}\left(1 \pm \rho_{p^{\prime}}\right)\left(1 \pm \rho_{k^{\prime}}\right)}{4 \sqrt{(p \cdot k)^{2}-m_{p}^{2} m_{k}^{2}}} .
$$

Comparing Eqs. (2.1) and (2.2) yields

$$
\eta=\left[\frac{d^{3} p}{(2 \pi)^{3} p^{0}}\right]\left[\frac{d^{3} k}{(2 \pi)^{3} k^{0}}\right] \sqrt{(p \cdot k)^{2}-m_{p}^{2} m_{k}^{2}} \rho_{p} \rho_{k} d \sigma .
$$

To get the rate of collision per unit time $\eta^{(1)}$ of one $p$-particle in the plasma, we divide $\eta$ by $\rho_{p} d^{3} p /(2 \pi)^{3}$, the volume density of $p$-particles:

$$
\eta^{(1)}=\frac{d^{3} k}{(2 \pi)^{3} p^{0} k^{0}} \sqrt{(p \cdot k)^{2}-m_{p}^{2} m_{k}^{2}} \rho_{k} d \sigma .
$$

To calculate the mean free path associated with the above process, we need to find the total rate of collision per unit time $\eta_{t o t}^{(1)}(p)$ for one particle with initial 4-momentum $p$ into any final state in the allowed phase space, using the total cross section $\sigma$. From Eqs. (2.2) and (2.4) we get

$$
\eta_{t o t}^{(1)}(p)=\frac{2}{(4 \pi)^{5}} \int \frac{d^{3} k}{p^{0} k^{0}} \int \frac{d^{3} p^{\prime} d^{3} k^{\prime}}{p^{\prime 0} k^{\prime 0}} \delta^{(4)}\left(p+k-p^{\prime}-k^{\prime}\right)|\mathcal{M}|^{2} \rho_{k}\left(1 \pm \rho_{p^{\prime}}\right)\left(1 \pm \rho_{k^{\prime}}\right) .
$$

Note that each scatterer included will give an additive contribution of this form to $\eta_{t o t}^{(1)}$.

The collision time $\tau(p)$, the length of time between two successive collisions for a $p$ particle, is the inverse of $\eta_{t o t}^{(1)}(p)$

$$
\tau(p)=\frac{1}{\eta_{t o t}^{(1)}(p)}
$$


The distance $l(p)$ such a $p$-particle travels between two collisions is then given by $l(p)=$ $\left(|\vec{p}| / p^{0}\right) \tau(p)$. We finally get the mean free path $\lambda$ for the $p$-particle by taking the thermal average of $l(p)$, using the thermal distribution of the $p$-particles. We thus get

$$
\lambda=\left[\int \frac{d^{3} p}{(2 \pi)^{3}} \rho_{p}\right]^{-1} \int \frac{d^{3} p}{(2 \pi)^{3}} \rho_{p} \frac{|\vec{p}|}{p^{0} \eta_{\text {tot }}^{(1)}(p)},
$$

where we have used Eq. (2.6). Note that this mean free path vanishes if the cross section suffers from infrared divergences. However, for diffusive processes, these divergences are suitably regulated and only processes with nontrivial momentum transfer contribute. The resulting mean free path (2.7) can then be related to the diffusion constant $D$ by the relation

$$
D=\frac{1}{3} \lambda \bar{v},
$$

where $\bar{v}$ is the mean velocity of the diffusing particle.

\section{RESULTS AND DISCUSSION}

We begin this section by describing some of the thermal properties of gluons in the plasma and how we incorporate these properties into our calculations. Due to interactions with the plasma, gluons develop temperature dependent masses. The longitudinal gluons

have a thermal Debye mass $m_{g}(T)=\sqrt{8 \pi \alpha_{s}} T$, where $\alpha_{s}=g_{s}^{2} /(4 \pi)$, at the 1-loop level and the transverse gluons have a non-perturbative thermal magnetic mass $m_{t}(T)$ that is zero at the 1-loop level and is expected to be $\mathcal{O}\left(g_{s}^{2} T\right)$. Thus, we may assume that the infrared screening of longitudinal gluons occurs at a momentum scale $m_{g}$, and the similar scale for the transverse gluons is likely lower. At the electroweak phase transition temperature $T_{c} \approx 100$ $\mathrm{GeV}, \alpha_{s} \approx 0.1$ and $m_{g} \approx 1.6 T$. Since the magnetic mass is unknown, the choice of transverse infrared momentum cutoff is rather arbitrary. However, because $g_{s} \approx 1$ at scale $T_{c}$, it is reasonable to assume that $m_{t}$ is of order $T$. In Table [, we take as two representative values $m_{t}=T$ and $m_{t}=m_{g}$.

The amplitude for the $t$-channel squark-quark diagram of Fig. [ is 


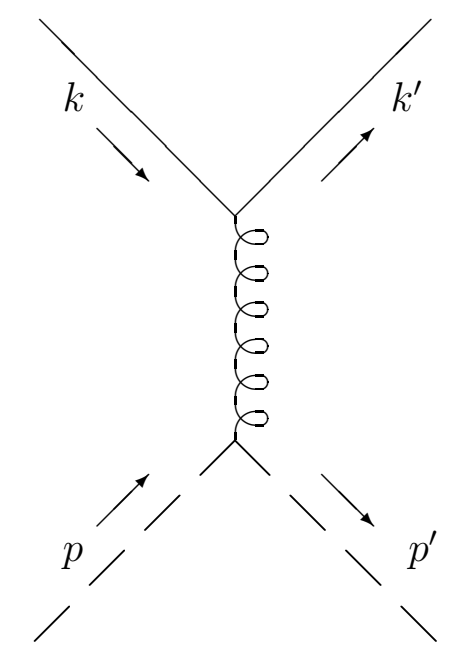

FIG. 1. t-channel squark-quark Feynman diagram.

$$
\mathcal{M}_{s q}=-8 \pi \alpha_{s} T^{a} p^{\mu} D_{\mu \nu} \bar{q}\left(k^{\prime}\right) T^{a} \gamma^{\nu} q(k)
$$

where $T^{a}$ is a generator in the adjoint representation of the $S U(3)_{c}$ color gauge group, and $q$ is a quark spinor. We work in the Landau gauge where, as explained in the appendix, $\left(k^{\prime}-k\right)^{\mu} D_{\mu \nu}=0$. Since $p+p^{\prime}=2 p+k-k^{\prime}, \mathcal{M}_{s q}$ does not depend on $p^{\prime}$ explicitly. We use the amplitude $\mathcal{M}_{s q}$ of Eq. (3.1) to estimate the squark mean free path $\lambda_{s}$ and diffusion constant $D_{s}$ from Eqs. (2.7) and (2.8). The amplitude is squared and summed over all 72 species of quark scatterers (3 colors, 2 spins, 6 flavors, and antiparticles). We have numerically computed $\lambda_{s}$ and $D_{s}$ for supersymmetry breaking squark masses ranging from $50 \mathrm{GeV}$ to $200 \mathrm{GeV}$ and observed that their mass dependence is weak. Our results are presented in Table I, where the values of $\lambda_{s}$ and $D_{s}$ have been computed for $m_{s}=100 \mathrm{GeV}$.

In Ref. [6], $D_{q}$ is computed using only the $t$-channel quark-quark scattering amplitude of Fig. 2, given by

$$
\mathcal{M}_{q q}=-4 \pi \alpha_{s} \bar{q}\left(p^{\prime}\right) T^{a} \gamma^{\mu} q(p) D_{\mu \nu} \bar{q}\left(k^{\prime}\right) T^{a} \gamma^{\nu} q(k)
$$

To compare our method with that of Ref. [6], we have computed $D_{q}$, again numerically, using only $\mathcal{M}_{q q}$ (and again summing over 72 species of scatterers). The entries in Table [ 


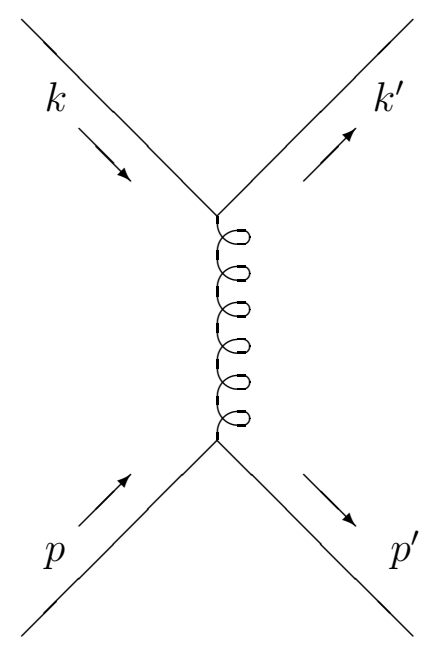

FIG. 2. $t$-channel quark-quark Feynman diagram.

labeled "JPT" refer to the numbers we get for the set of parameters that are used in Ref. [6], namely $\alpha_{s}=1 / 7$ and $m_{g}=m_{t}=\sqrt{8 \pi \alpha_{s}} T=1.9 T$. We see that using the JPT parameters, we obtain the estimate $D_{q} \sim 6 / T$ of Ref. [6], where only the leading logarithmic contributions were considered. This suggests that our momentum randomization approximation, where the momentum transfer $q \gtrsim T$, yields reasonable estimates for the diffusion constant. However, we also note that in Ref. [6] the diffusion constant $D \sim 1 /\left[g_{s}^{4} \ln \left(g_{s}^{-1}\right)\right]$, where the logarithmic dependence comes from the use of a $g_{s}$ dependent $m_{g}$ as the infrared regulator. In our approach, as mentioned before, for physical values of $g_{s}$, we can use $m_{g}$, and perhaps $m_{t}$, as the infrared cutoffs, but in general, the cutoff we choose is not a function of $g_{s}$, and does not vanish as $g_{s} \rightarrow 0$, for it has to be chosen to satisfy $q \gtrsim T$. In this way, our computations will yield $D \sim 1 /\left(g_{s}^{4}\right)$, which represents the $\alpha_{s}$ dependence of the amplitudes (3.1) and (3.2). Therefore, the behavior of our results differs from those of Ref. [6] by $1 / \ln \left(g_{s}^{-1}\right)$. However, for physical values of $g_{s}$, the logarithm is of order unity. Taking the result of Ref. [6] as a fair estimate of $D_{q}$, we believe that our results are reliable up to factors of order unity. Note that we do not consider all the processes that contribute at this level: scatterings from on-shell gluons in the plasma also provide a substantial contribution. However, the results 


\begin{tabular}{|c|c|c|c|c|c|c|}
\hline & $\alpha_{s}$ & $m_{g}$ & $m_{t}$ & $\lambda$ & $D$ & Remarks \\
\hline \multirow[t]{3}{*}{ quark } & $1 / 10$ & $1.6 T$ & $T$ & $13 / T$ & $4 / T$ & \multirow[b]{3}{*}{ JPT } \\
\hline & $1 / 10$ & $1.6 T$ & $1.6 T$ & $24 / T$ & $8 / T$ & \\
\hline & $1 / 7$ & $1.9 T$ & $1.9 T$ & $18 / T$ & $6 / T$ & \\
\hline \multirow{3}{*}{$m_{s}=100 \mathrm{GeV}$} & $1 / 10$ & $1.6 T$ & $T$ & $12 / T$ & $3 / T$ & \multirow[b]{3}{*}{ JPT } \\
\hline & $1 / 10$ & $1.6 T$ & $1.6 T$ & $18 / T$ & $5 / T$ & \\
\hline & $1 / 7$ & $1.9 T$ & $1.9 T$ & $14 / T$ & $4 / T$ & \\
\hline
\end{tabular}

TABLE I. Results for $\lambda$ and $D$

of Ref. [7] suggest that the inclusion of these diagrams will not change the results by more than a factor of 2 .

Our calculations suggest that $\lambda_{s} \approx \lambda_{q}$ and $D_{s} \approx D_{q}$, up to factors of order unity, and most likely to within $30 \%$, and that the effects of different statistics, masses, and couplings on the values of $D$ and $\lambda$ for squarks and quarks are not strong. For $m_{t}=T<m_{g}$, we roughly get $\lambda \lesssim 10 / T$ and $D \lesssim 3 / T$. On the other hand, if $m_{t}=m_{g}$, our results increase by about a factor of 2 .

Electroweak baryogenesis scenarios that use quarks or squarks for charge transport in a first order phase transition suffer from a suppression due to decoherence that is caused by multiple scatterings of these strongly interacting particles across the width of the expanding Higgs wall. A measure of the strength of decoherence is the ratio of the mean free path of the particles to the width $w$ of the wall [5]. In Ref. [10], a 2-loop MSSM calculation of the Higgs wall profile gives $w \approx 25 / T$. Using this value of $w$, our results yield $\lambda / w \lesssim 1 / 2$, which suggests that although the effects of decoherence are not negligible, they are not severe.

\section{ACKNOWLEDGEMENTS}

It is a pleasure to thank Ann Nelson, John Preskill, Krishna Rajagopal, Dam Son, Mark Wise, and Laurence Yaffe for insightful discussions. We would also like to thank Martin 
Gremm and Iain Stewart for their helpful comments. This work was supported in part by the U.S. Dept. of Energy under Grant No. DE-FG03-92-ER40701.

\section{APPENDIX: THERMAL GLUON PROPAGATOR}

In this appendix, we give the expression we use for the approximate thermal gluon propagator in a plasma, taking the different properties of the longitudinal and transverse gluons into account as represented by their respective cutoffs $m_{g}$ and $m_{t}$. Let $n^{\mu}=(1,0,0,0)$ be the 4 -velocity of the plasma in the plasma frame. We denote the 4-momentum $q$ of the propagating gluon by $q^{\mu}=\left(q^{0}, \vec{q}\right)$ in the plasma frame. The component of $n$ that is orthogonal to $q$ is given by $\tilde{n}$, where

$$
\tilde{n}^{\mu}=n^{\mu}-\frac{(n \cdot q) q^{\mu}}{q^{2}}
$$

We define two projection operators $P_{\mu \nu}$ and $Q_{\mu \nu}$, where

$$
P_{\mu \nu}=g_{\mu \nu}-\frac{q_{\mu} q_{\nu}}{q^{2}}-\frac{\tilde{n}_{\mu} \tilde{n}_{\nu}}{\tilde{n}^{2}}
$$

and

$$
Q_{\mu \nu}=\frac{\tilde{n}_{\mu} \tilde{n}_{\nu}}{\tilde{n}^{2}}
$$

The expression for the Landau gauge thermal gluon propagator in our approximation is then given by

$$
D_{\mu \nu}^{(L)}=-\left[\frac{1}{q_{T}^{2}} P_{\mu \nu}+\frac{1}{q_{L}^{2}} Q_{\mu \nu}\right],
$$

where $q_{T}^{2}=q^{2}-m_{t}^{2}$ and $q_{L}^{2}=q^{2}-m_{g}^{2}$, and from $q^{\mu} P_{\mu \nu}=q^{\mu} Q_{\mu \nu}=0$, we have $q^{\mu} D_{\mu \nu}^{(L)}=0$. 


\section{REFERENCES}

[1] A. E. Nelson, D. B. Kaplan, and A. G. Cohen, Nucl. Phys. B373, 453 (1992);

A. G. Cohen, D. B. Kaplan and A. E. Nelson, Phys. Lett. B245, 561 (1990); Nucl. Phys. B349, 727 (1991); Phys. Lett. B294, 57 (1992); B336, 41 (1994).

[2] P. Huet and A. E. Nelson, Phys. Lett. B355, 229 (1995); Phys. Rev. D53, 4578 (1996).

[3] M. Carena, M. Quirós and C. E. M. Wagner, Phys. Lett. B380, 81 (1996);

D. Delepine, J.-M. Gerard, R. Gonzalez Felipe, J. Weyers, Phys. Lett. B386, 183 (1996);

J. R. Espinosa, Nucl. Phys. B475, 273 (1996);

D. Bödeker, P. John, M Laine, and M.G. Schmidt, Nucl. Phys. B497, 387 (1997);

B. de Carlos and J. R. Espinosa, hep-ph/9703212; M. Carena, M. Quirós, A. Riotto, I. Vilja and C. E. M. Wagner, Nucl. Phys. B503, 387 (1997);

M. Carena, M. Quirós, C. E. M. Wagner, hep-ph/9710401.

[4] A. Riotto, hep-ph/9709286.

[5] H. Davoudiasl, K. Rajagopal, and E. Westphal, hep-ph/9707540, to appear in Nucl. Phys. B.

[6] M. Joyce, T. Prokopec and N. Turok,Phys. Rev. D53, 2930 (1996); ibid. 2958.

[7] G. D. Moore and T. Prokopec, Phys. Rev. D52, 7182 (1995).

[8] H. A. Weldon, Phys. Rev. D26, 1394 (1982).

[9] E. Braaten, R. D. Pisarski, Phys. Rev. D42, 2156 (1990);

E. Braaten, R. D. Pisarski, Nucl. Phys. B337, 569 (1990).

[10] J. M. Moreno, M. Quirós, and M. Seco, hep-ph/9801272. 